\title{
The Importance of Rigorous Methods in a Growing Research Field: Five Practices for ASMR Researchers.
}

\author{
Thomas J. Hostler \\ Manchester Metropolitan University, UK
}

\begin{abstract}
A rigorous field of research is constructed on reproducible findings that allow researchers to confidently formulate hypotheses and build theories from accessible literature. As a nascent area of research, the study of Autonomous Sensory Meridian Response (ASMR) has the opportunity to become such a field through the adoption of transparent and open research practices. In this paper I outline five such practices that can help achieve this aim: Preregistration, sharing data and code, sharing materials, posting preprints, and collaboration. Failing to adopt such principles could allow the proliferation of findings that are irreproducible and delay the progress of the field.
\end{abstract}

Keywords: Autonomous Sensory Meridian Response, ASMR, Open Science, Preregistration

\section{Background}

Autonomous Sensory Meridian Response (ASMR) is a sensory experience characterized by a tingling sensation on the crown of the head and feelings of calmness, relaxation, and altered consciousness. The experience is triggered by audiovisual stimuli commonly found in popular online YouTube videos including whispering, tapping and scratching sounds, close personal attention, and expert hand movements. People seek out and watch these videos to experience ASMR, which produces self-reported psychological benefits including reducing stress, anxiety, depression, loneliness, and insomnia (Barratt \& Davis, 2015). In May 2022, 'ASMR' was the 3rd most popular search term on YouTube in the world, with nearly 15 million searches (Hardwick, 2022).

Despite huge public popularity, ASMR has only recently become the subject of scientific enquiry. First described in academic literature by Ahuja (2013), the first empirical investigation into ASMR was published by Barratt and Davis (2015), an online survey describing the phenomenon, common triggers and reasons for engaging with ASMR content. Since then, the number of published papers on ASMR has increased year on year (Figure 1), indicating a growing academic interest in the phenomenon. Researchers have subsequently investigated the triggers of ASMR (Barratt et al., 2017), the physiological concomitants (Poerio et al., 2018), personality correlates and co-occurrences with other sensory experiences (Bedwell \& Butcher, 2020; Fredborg et al., 2017, 2018; Keizer et al., 2020; Lee et al., 2019; McErlean \& Banissy, 2017, 2018; McErlean \& OsborneFord, 2020), underlying brain regions (Lochte et al., 2018; Smith et al., 2019a; Smith et al., 2019b; Smith et al., 2017), and developed reliable self-report measures (Roberts et al., 2019) and curated stimuli sets (Liu \& Zhou, 2019).

As a new field of study, there are countless novel directions open for ASMR researchers to take. This may lead to an incentive to conduct and publish research quickly in order to become the first to set foot 


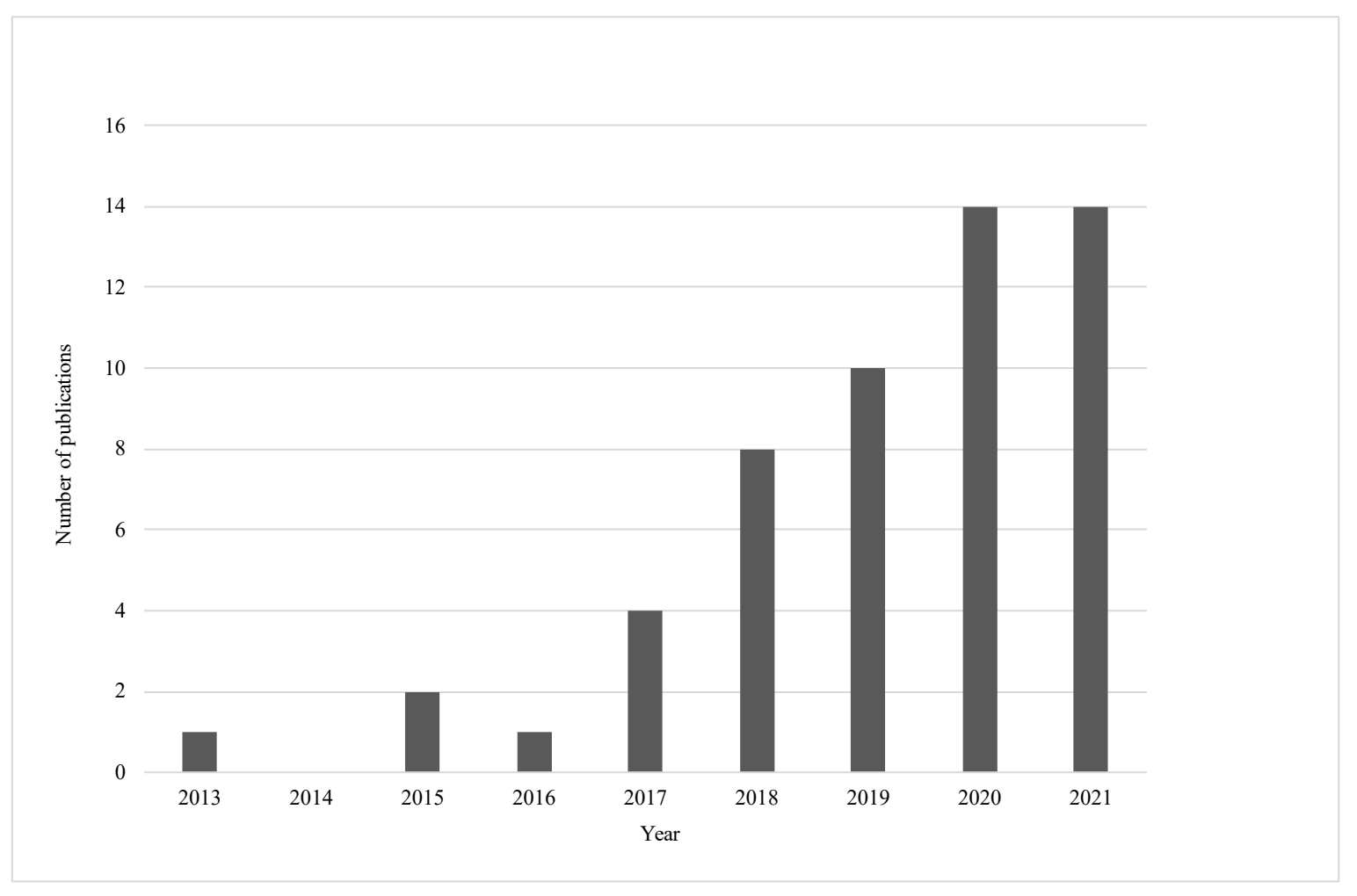

Figure 1. Number of publications published per year about "Autonomous Sensory Meridian Response" from 2013 2021, as indexed on Web of Science.

on an untouched and fertile soil of scientific discovery. It is well documented that such publishing incentives can blind researchers to biases that encourage the overinterpretation of data and the use of questionable research practices (Giner-Sorolla, 2012; Higginson \& Munafò, 2016). In turn, this leads to an accumulation in the literature of spurious results, inaccurate estimates of effect sizes, and findings that are irreproducible (Ioannidis, 2005; Munafò et al., 2017).

This is a particular concern for a new and developing field, where a 'hard core' of replicated and accepted findings have yet to be established (Lakatos, 1978). To develop such a core, and allow researchers to confidently build upon the findings of previous research, it is paramount that such findings must be obtained using unbiased and rigorous practices and that these methods are clear and transparent to allow others to replicate methodologies as closely as possible. Therefore, adopting transparent and rigorous research practices will accelerate the accumulation of 'established' ASMR find- ings, and subsequently, theory-building and the field at large. Conversely, utilising traditional 'closed' research practices is likely to facilitate the publication of findings and results that do not replicate (Smaldino \& McElreath, 2016), thus leading future researchers to pursue unproductive lines of inquiry and delaying the development of the field. It is theorised that developing fields go through three stages of differentiation, mobilisation, and legitimacy building (Hambrick \& Chen, 2008). ASMR research has a clear unique focus, which helps to differentiate it from the study of other sensory experiences. However, it currently lacks mobilisation in terms of organisation and resources, and legitimacy in terms of rigorous methodologies and compelling evidence. Hambrick and Chen (2008) argue that the growth and legitimacy of a field depends on the quality of the research produced: "Scholars in more established fields will look for indicators that the new area's research resembles a style they hold in high regard, a style that is 'on the right track." 
In this paper, I outline five practices that ASMR researchers can use to improve the legitimacy of the field, accelerate the mobilisation of the research community, and increase the transparency, rigour, and reproducibility of their research: Pre-registration, sharing data and code, sharing study materials, preprints and post-prints, and collaboration. For each, I will explain their applicability to ASMR research with examples.

\section{Pre-Registration}

Pre-registration is the process of publicly and transparently reporting a study's design and analysis plan prior to data collection (Nosek et al., 2018). It typically takes the form of a time-stamped online document that details a study's design, hypotheses, sampling plan, and data processing and analysis plan. The purpose of preregistration is to preclude the use of questionable research practices including ' $p$-hacking' (exploiting undisclosed flexibility in data analysis plans to obtain significant results) and 'HARKing' (Hypothesizing After the Results are Known to present such significant findings as predicted a priori) (Chambers, 2017).

This does not mean that studies that were not preregistered have definitely engaged in $p$-hacking, or trying multiple analyses until a significant result is obtained, but that it is impossible to tell. Only by reporting one's analysis plan in advance can someone say with confidence that there was no undisclosed flexibility in the analysis. It is important to note that $p$ hacking is often unconscious and the result of human, fallible researchers operating with cognitive biases and within a career progression framework that incentivises publication of significant results. Faced with a multitude of analytical decisions to take regarding participant exclusion, data quality, and questionnaire scoring, researchers analysing data without a pre-defined plan end up walking in a "garden of forking paths" (Gelman \& Loken, 2019), with each path leading to a different statistical outcome. The combination of the ease of posthoc rationalisation of findings, confirmation bias, and the incentives to discover and publish significant results mean that researchers are likely to publish and justify analyses that turn out as significant, even though alternative analyses may have produced different findings (Chambers, 2017). A hypothetical example below using ASMR research illustrates this, where two research teams in alternate universes each recruit 250 ASMR participants and administer them with (amongst other things) the ASMR-Checklist (Fredborg et al., 2017) and the "Poor attentional control" subscale of the Short Imaginal Process Inventory (SIPI) (Huba et al., 1982). Both research teams obtain exactly the same dataset, and test exactly the same hypothesis: that there is a re- lationship between ASMR and attentional control. The first research team, Anna and Abel, proceed with data analysis as follows:

When analysing their data, they decide to exclude 10 people who report in a qualitative response that they experience atypical ASMR (e.g. they only experience it in response to real-world triggers), in order to get a more homogenous sample of only those who get a typical ASMR experience from videos. When scoring the SIPI, they follow the instructions from Huba et al (1982) and begin with a baseline score of 42, and add/subtract the scores of specific questions to produce an overall score. They notice the distribution of the SIPI scores are non-normal due to skewness and a number of extreme scores. They follow textbook instructions to use a non-parametric test for the analysis. They find no significant relationship between ASMR and Poor Attentional Control (rho $=-.135, p=$ .091) and conclude that there is not sufficient evidence from the study to say that the constructs are related. In their discussion, they suggest future research may want to focus on alternative constructs.

In the alternate universe, two other researchers (Brett and Bushra), test the same hypothesis with the same dataset, but in a different way:

When analysing their data, they decide to keep in those who report that they experience atypical ASMR, as they think that ASMR is probably quite a heterogenous experience overall and they do not want to make a judgement on what 'counts' as ASMR. However, when scoring the ASMRchecklist, they decide to exclude the trigger of "watching someone apply makeup" from the scoring, as they find a relatively low response rate for this trigger, and believe it could be unduly influenced by gender and cultural norms. When scoring the SIPI, they use a traditional scoring method of recoding negative items and computing a mean score of the items. They notice the distribution of the SIPI scores are non-normal due to skewness and a number of extreme scores. They follow textbook instructions to log-transform the data before analysis. They find a significant negative relationship between ASMR and Poor Attentional Control $(r=-.195, p=.035)$ and conclude the constructs are related. In their discussion, they suggest future research should explore this relationship further.

In both of these examples, there were a number of decisions the researchers had to take regarding the analysis: who to exclude, how to score the questionnaires, which analysis to use. Many of these decisions were data-driven: Brett and Bushra would not have decided to remove that specific trigger from the checklist scoring 
until seeing the relatively low score. All of these decisions were reasonable and justified, but done so posthoc. The particular decisions made led to different conclusions. In both these cases, the eventual $p$-value was accepted and interpreted as evidence for or against their pre-planned hypothesis, and the finding incorporated into the literature. Due to the small existing literature base and lack of current theory, it would not be difficult to come up with plausible explanations of why ASMR may (or may not) be related to poor attentional control, and to suggest diverging directions for future research. However, the different 'paths' taken by the researchers illustrate that despite testing the same a priori hypothesis, their analyses were essentially exploratory, meaning that it would be wrong to draw any firm conclusions from the data.

Pre-registering analyses means that researchers have to think more carefully in advance about their hypotheses, and justify analysis decisions in advance: What 'counts' as ASMR for the research question I'm interested in? What criteria for data quality am I willing to accept? What are the psychometric properties of my questionnaire and is it suitable to answer the research question? Answering these questions may be difficult but not impossible with a bit of thought and knowledge of the literature and good methodological practice. One concern many researchers have with preregistration is that it will prevent them from running exploratory tests, or will constrain their analysis. What if the data turns out nothing like they thought and they cannot run the test they preregistered? This concern is understandable but ill-founded. Pre-registration does not prevent researchers from performing or reporting non-preregistered analyses, or testing hypotheses that they thought of at a later date. However, it necessitates the accurate reporting of such tests as exploratory, and with it the implications for the lack of 'hard' inferences that can be drawn from the data. The commonly used procedure of Null Hypothesis Significance Testing (NHST) means that a proportion of 'significant' results are expected by chance. As exploratory analyses are by definition a search for potentially 'chance' findings, conclusions drawn from exploratory tests need to be replicated in order to add value to the literature. On the flip side, a statistical test that supports a pre-registered hypothesis allows one to draw much more confident inferences from the results and provides a much sturdier finding upon which to build. Studies can be pre-registered on websites including AsPredicted (http://aspredicted.org) or the Open Science Framework (OSF) (http://osf.io). An example of a preregistration for an ASMR study (Poerio et al., 2022) can be found here: https://osf.io/pjq6s which we are happy for other researchers to use as a guide, although do not claim that it is a perfect example. O. Klein et al. (2018) present a useful guide for writing pre-registrations.

Registered Reports. A recent initiative in scientific publishing that utilises the idea of pre-registration for maximum benefit is the Registered Report (RR) (Chambers \& Tzavella, 2020). An RR is a specific type of article, which is submitted to a journal prior to data collection. A researcher will outline the background and idea for their research, and submit this along with their methodology and pre-registration for the study they wish to run. At this point, the study goes out for peer review, and may return with revisions if necessary. Once the revisions have been satisfied, the journal will grant the paper "in principle acceptance", meaning they guarantee to publish the subsequent results of the study, regardless of the outcomes. RRs therefore combine the methodological rigor of a pre-registered study with a commitment to publish null results if necessary, thus correcting for publication bias in the literature. Given that publication bias can severely hamper progress in a psychological field (Ferguson \& Heene, 2012), the widespread use of RRs in the new field of ASMR research would help to prevent the problem of publication bias occurring in the first place. At the time of writing, journals that currently or will soon offer RRs that are likely to be open to publishing research on ASMR include: Affective Science; Attention, Perception, \& Psychophysics; Cognition and Emotion; Consciousness and Cognition; Experimental Psychology; Frontiers in Psychology; International Journal of Psychophysiology; iPerception; Journal of Personality and Social Psychology; Nature Human Behaviour; Neuroscience of Consciousness; Perception; PLOS ONE and Psychological Science. A full live list of journals that accept RRs can be found at: https://cos.io/rr/

\section{Sharing Data and Code}

As well as making data analysis decisions transparent via pre-registration, researchers should also share the data itself, and the analysis code used to produce the results. 'Sharing' in this context means making the data available on an accessible online repository, rather than authors responding to ad hoc requests to share data via email. Empirical research (and the personal experience of anyone who has done a meta-analysis) confirm that sharing data on an ad hoc basis is highly ineffective for a number of reasons including poor record keeping and authors changing institutions, and availability declines rapidly over time (Savage \& Vickers, 2009; Vines et al., 2014; Wicherts et al., 2011; Wicherts et al., 2006).

Making data readily available has multiple benefits for cumulative science. The first is that when data is 
made available, other researchers can check whether they agree with the conclusions drawn from the data by conducting their own analyses. This has already been evidenced in ASMR research: We (Hostler et al., 2019) reinterpreted the data from Cash et al. (2018) study by accessing the raw dataset and visualizing it in a different way than presented in the original paper. By doing this, we came to a different conclusion and therefore attempted to 'correct' the literature in the spirit of the selfcorrecting mechanism of science (Merton, 1974). This would not have been possible without access to the raw data.

The second benefit of providing raw data is that researchers can perform alternate analyses on the data that the original authors did not or could not, to maximise the utility of the original research. It is impossible to know what other researchers might find interesting or need to know about the data, and therefore not possible to know which statistics and analyses would need to be reported. A hypothetical example is that of a future meta-analyst who wishes to compute an overall effect size from past experimental ASMR research. Following the guidelines of Lakens (2013), they find that in order to compute an accurate effect size from previous research utilising within-subjects designs, they need information about the correlation ( $r$ ) between the two repeated measures. This statistic is very rarely reported in studies, but by making the raw data available, it doesn't have to be: the meta-analyst can download the data and calculate it themselves. Another example is that of McErlean and Osborne-Ford (2020) who were able to access the open data of Fredborg et al. (2018) and perform additional analyses to facilitate the comparison of their findings.

A third benefit concerns the sharing of analysis code. This allows the computational reproducibility of the findings and is an important mechanism for error detection. By uploading the code used to produce the findings used in the paper, other researchers can see exactly 'which buttons were pressed' to reach the final numbers reported in the paper, and whether the preregistration was followed (if used). To maximise accessibility, researchers should try to use non-proprietary analysis software such as $R$, so that any researcher has the potential capacity to produce the analysis, regardless of institutional access to proprietary programs such as SPSS or Stata. The 'gold standard' would be to use online container technology to recreate the virtual environment in which the original code was executed, in case different versions of software produce different results (see Clyburne-Sherin et al., 2018). If this is not possible, then researchers should still share analysis code from proprietary programs such as SPSS via the syntax function: Partial accessibility is better than none.

Data sharing is becoming increasingly common across psychology in general, thanks in part to journal and funder requirements (Houtkoop et al., 2018) and a few current examples in ASMR research have been highlighted above. The challenge for the future is to ensure that data sharing in ASMR research is done consistently, and in an ethical and thoughtful way that maximises its benefit, not merely as an afterthought to meet journal requirements. Table 1 highlights some potential issues for sharing data and code in ASMR research and some possible solutions. For more useful guidance see Meyer (2018).

\section{Sharing study materials}

One of the most important transparent research practices for ASMR research is the sharing of study materials, such as ASMR videos. In experimental designs, researchers will often want to use ASMR videos to induce ASMR in order to measure the ASMR response and any influences on this. In correlational research, researchers may want to use ASMR videos to verify the ASMR status' of participants and confirm whether they experience the sensation (Hostler et al., 2019). In order to improve consistency amongst studies and the reproducibility of findings, it is important to know exactly which videos are used in ASMR research for these purposes.

Most researchers will take ASMR videos created by ASMRtists (content creators) from YouTube, rather than create original ASMR content themselves. Whilst the use of these videos in research itself is likely to fall under 'fair use' copyright laws, it is unclear whether sharing the original video files on other public repositories may infringe copyright. Depending on the number and length of videos, it may also be prohibitive due to the large file sizes. Researchers can circumvent this issue by sharing information on the original sources of the videos used, including URLs and timestamps (for an example from Poerio et al. (2018) see https://osf.io/u374f/). Researchers should also cite the exact video number used from stimuli sets such as Liu and Zhou (2019), and include descriptions about the content of the videos used in the manuscript or supplementary materials, in case of broken links.

As ASMR research is in an infant stage, there is currently little knowledge about how the specific content and triggers in an ASMR video may affect the ASMR response. However, this is the sort of question that could prove to be important in future systematic reviews of ASMR research-if sufficient information is available to investigate it. Ensuring that the exact content of videos used in ASMR research is accessible will allow future 
Table 1

Issues and solutions for data sharing in ASMR research.

\begin{tabular}{ll} 
Issue & Solution \\
\hline Ethical concerns & Most data from ASMR experiments are unlikely to be particularly \\
& sensitive, but in some cases researchers may collect data that ne- \\
& cessitates increased anonymity (e.g., data on mental health diag- \\
& noses), via the removal of potentially identifying information (e.g. \\
& age and gender). Participants must also consent for their data to \\
& be shared, so researchers should be careful to avoid claiming that \\
& data will be "kept confidential" or "destroyed" in their ethics doc- \\
& umentation and participant information sheets. \\
\hline Data comprehensibility & Shared data is only useful if other researchers can interpret it. \\
& Researchers should include supplementary "data dictionary" files \\
& alongside data files, to explain what each variable represents, and \\
& how any categorical variables have been coded. \\
\hline Data accessibility & Where possible, data should be provided in file formats for non- \\
& $\begin{array}{l}\text { proprietary software, for example .CSV files. Ideally, syntax or } \\
\text { data analysis code should include any pre-processing of data, in- } \\
\text { cluding scoring questionnaires and removing outliers, as well as } \\
\text { analyses. File names should be machine readable, utilising un- } \\
\text { derscores rather than spaces, and include information about what } \\
\text { the file contains (e.g. "ASMR_Study1_EEGData.csv") }\end{array}$ \\
\hline $\begin{array}{l}\text { Data and analysis code files can often be hosted as supplemen- } \\
\text { tary files on journal websites, or institutional data repositories. }\end{array}$ \\
$\begin{array}{l}\text { However, hosting the data on a searchable repository such as } \\
\text { the Open Science Framework (http://osf.io), Figshare (http:// } \\
\text { figshare.com) or Zenodo (http://zenodo.org) can increase discov- } \\
\text { erability and utility, as well as allowing the researcher greater con- } \\
\text { trol over updating the file in future, if necessary. }\end{array}$ \\
\hline Data storage
\end{tabular}

meta-analysists to code for any number of factors - for example, which triggers are present; whether the ASMRtist is male or female - as moderating factors in analyses of ASMR effects.

In addition to sharing ASMR video materials used in research, researchers should utilise external repositories such as the Open Science Framework to share all their study materials including recruitment media, participant instructions, questionnaires, and flow-chart protocols. Whilst journal articles often have strict word limits on the amount of information that can be included in a method section, there are no such limits to uploading supplementary material in separate repositories, to which the DOIs can be linked to in the article itself. Sharing materials is one of the easiest transparency initiatives to introduce into a research workflow. For example, an entire online questionnaire created in Qualtrics can be downloaded as a .pdf and uploaded to the OSF with only a few clicks. The benefit is that other researchers can see exactly what information participants were told, how the study was set up, and which questions were asked, to allow the study to be replicated as closely as possible.

\section{Preprints \& Post-prints}

The traditional publishing workflow dictates that the findings of a piece of research are only communicated to other scientists and the public after undergoing peer review when the study is published as a journal article. Preprints are online copies of manuscripts that shortcut this process, as they are uploaded to a repository prior to peer review and journal submission (Speidel \& Spitzer, 2018). Given that peer review can often take several months before a study is published, preprints accelerate the dissemination of research findings, and thus the productivity and development of the entire field. Preprints also increase the visibility and discoverability of research, which can facilitate networking. They can also be used to communicate research that would otherwise sit in a "file drawer", for example studies with null results, small sample sizes, or undergraduate projects (Sarabipour et al., 2019). Although researchers are encouraged to check individual journal policies, most journals do not regard preprints as 'prior publication' and 
so they do not act as a disqualifier for eventual journal publication.

One common concern regarding preprints is that it is believed posting the results of findings online before publication could allow a researcher to be 'scooped' and their idea stolen. However, preprints are associated with a permanent DOI meaning that they have an established mark in the scientific record, and are indexed by Google Scholar and CrossRef. Therefore, posting a preprint can actually allow a researcher to indicate that they recorded a finding or idea first, without having to wait for 'official' publication. Practically speaking, it is also unlikely that another lab would have the time to run an entire study, write up the results, and submit and get it accepted for publication, in the time it takes for a posted preprint to be peer reviewed.

Most psychology researchers submit their preprints to the dedicated psychology preprint server PsyArXiv (https://psyarxiv.com/) but ASMR research could also be hosted on the server for "mind and contemplative practices" research, MindRXiv (https://mindrxiv.org/), bioRXiv for biological mechanisms (https://biorxiv.org) or a geographical server such as AfricaRXiv (https://osf. io/preprints/africarxiv/).

\section{Post-prints}

Confusingly, the term 'preprint' is sometimes also used to refer to a manuscript uploaded after publication, to circumvent publisher paywalls. These 'postprints' are a common way to allow for 'Green' Open Access, where authors can upload a non-typeset copy of their article to their personal, institutional, or academic social networking webpage (e.g. http://researchgate. net; http://academia.edu) if their actual article is not published Open Access in the journal itself. Sharing post-prints of articles is an increasingly common requirement of publicly funded research, and so it is likely that many ASMR researchers already do this. However, it is worth reiterating that if an ASMR research article is not published 'Gold' Open Access, authors should try to ensure that the non-formatted manuscript is available in as many places as possible, to allow for maximum discoverability and use by the ASMR research community.

\section{Collaboration}

The final practice is a broader call for more collaborative research in ASMR, and for researchers to combine resources together in the spirit of 'team science' (Munafò et al., 2017) to conduct larger, well-powered investigations into the phenomena. Large, multi-lab studies are becoming increasingly common in psychological science (e.g. Camerer et al., 2018; Ebersole et al., 2016; R. A. Klein et al., 2014; R. A. Klein et al., 2018; Open
Science Collaboration, 2015) as researchers realise the benefits that pooling resources can have, particularly when it comes to participant recruitment, traditionally one of the more practically difficult aspects of psychological research.

Underpowered studies remain the norm in psychology (Maxwell, 2004) and researchers commonly overestimate the power in their own studies (Bakker et al., 2016). This is compounded by the fact that traditional methods of calculating sample size underestimate the number of participants needed, due to imprecise measures of effects: Taking effect sizes from previous studies at face value can underestimate the number of participants needed by a factor of ten (Anderson et al., 2017). As an example, when accounting for publication bias and Type 1 error, researchers wishing to replicate a published study finding a between-two-groups effect size of $d=0.68$ could require up to a total of $N=$ 662 to achieve power of .73. Whilst online research methods can facilitate collecting data from large samples like these, many researchers will want to conduct ASMR studies in the laboratory to control for environmental factors, standardize participant experience, or employ physiological or neuroscientific measures. However, the time and resources needed to collect large sample sizes in-person can make this sort of data collection prohibitive.

The solution is for researchers to work together to pool resources. Whilst it may be difficult for a single lab to recruit and test 600 participants in-person, it would be feasible for six labs to work together and collect 100 participants each for a single, high-powered study. Utilising the other principles of transparent research, the researchers involved could share materials and pre-register the design of the study together to ensure consistency in the set-up of the experiment and data collection. The data itself would then be shared and analysed together with a shared transparent analysis code, so that all contributors could see exactly how the final results were reached.

A relevant example of a large, multi-lab study investigating an emotional, sensory phenomenon is Zickfeld et al. (2019). Researchers from 23 different laboratories in 15 countries across five continents worked together to investigate "Kama Muta", the distinct emotional experience of 'being moved'. The KAVIAR Project (KAma muta Validity Investigation Across Regions) members worked together to standardise measurement of the phenomenon and construct a questionnaire, and then recruited a total sample of 3542 participants from across their respective labs. The study was pre-registered and conducted according to open research principles, and readers are encouraged to ex- 
plore the study OSF page as an exemplar of the organisation of a multi-lab project: https://osf.io/cydaw/. The resulting study provided convincing evidence of the validity of Kama Muta and the measurement tool used. As a new seminal paper on the topic, the study has already been cited 21 times in the literature.

Multi-lab collaborations of course require greater time and resources than individual lab studies, and come with unique challenges including integrating multiple datasets, multi-site ethical approval, translation issues, and the logistical headache of coordinating dozens of people in disparate time zones (Moshontz et al., 2018). In addition, traditional publishing incentives often favour quantity over quality. This means it is tempting for researchers to work in silos, working on their own studies as quickly as possible to increase their own number of publications and citations, to enhance their career.

However, as discussed earlier, this is bad for the field as a whole as it results in a multitude of underpowered and therefore non-replicable studies in the literature. This is not conducive to progress in the field, and it is a mistake to think that the limitations of small-sample individual studies and publication bias can be corrected post-hoc via meta-analysis (see Kvarven et al., 2019). The focus on quantity over quality is also a false economy for researchers interested in career-boosting metrics. By engaging in collaborative research, researchers will be producing high-value science: pre-registered, high-powered studies represent the highest quality of evidence on a topic, and so the results are inevitably published in more prestigious journals, receive greater prominence in the literature, and are cited more often. In addition, as a multi-lab study may have dozens (if not hundreds) of authors, each promoting the study through their own networks, social media channels, and at conferences and invited talks, the natural reach and visibility of the research is substantially increased. Concerns about author credit to large projects can be addressed by utilising clear contribution statements, such as the CRediT taxonomy (Holcombe, 2019), which ensures that all contributors receive appropriate recognition. Finally, as evidenced by the success of previous multi-lab studies, the practical barriers to large scale collaborations are far from insurmountable. Cloudbased document editing (e.g. Google docs; Dropbox Paper), multi-user conferencing and communication software (e.g. Zoom; Slack) and online project management software (e.g. Github; OpenProject) greatly ease the organisational burden of such initiatives and mean that barriers to multi-lab working are more often psychological than practical.

Researchers wishing to find other labs to collaborate with could utilise the StudySwap platform (https://osf. io/meetings/studyswap/), where labs can post "needs" or "haves" to facilitate sharing resources. Another option could be proposing an ASMR study to be conducted via the Psychological Science Accelerator (https: //psysciacc.org/), a network of psychology labs around the world who coordinate data collection on largescale studies. In addition, I invite ASMR researchers to sign up to a mailing list we have created for sharing ASMR research news and collaboration opportunities: https://asmr-net.us1.list-manage.com/subscribe? $\mathrm{u}=$ 503533eadcf849b8c108a79a7\&id=e0763fe0d0.

\section{Conclusion}

ASMR is a new and developing research field with enormous potential to inform our knowledge of emotion, sensory processing, and digitally-mediated therapies, as well as being a fascinating subject of study in its own right. The few studies conducted so far on ASMR have tentatively explored the phenomenon, and suggested exciting directions for research to pursue. However, in order for the field to develop and grow successfully, researchers need to be able to trust the findings in the literature and build theories and hypotheses upon 'stable' effects they are confident they will be able to replicate (Witte \& Zenker, 2017). Such a "progressive research program" only works when the results of hypothesis tests are trustworthy - i.e., free from bias (preregistered), able to be replicated (open materials), computationally reproducible and scrutinised for errors (open data and code), accessible (preprints) and come from high-powered studies (collaboration). Whilst there are also theoretical hurdles to overcome to advance research in this area, including questions to be answered around the definition and measurement of ASMR, transparency and collaboration are also a means for addressing these in a thorough and efficient manner (Boag et al., 2021). Witte and Zenker (2017) argue that psychology must "coordinate our research away from the individualistically organized but statistically underpowered short-term efforts that have produced the [replication] crises, toward jointly managed and well-powered long-term research programs". With the adoption of the open research practices outlined in this article, the nascent field of ASMR research has the potential to be the epitome of such a research program.

\section{Author Contact}

Please contact me via email (t.hostler@mmu.ac.uk) or twitter (@tomhostler); ORCID: https://orcid.org/ 0000-0002-4658-692X 


\section{Acknowledgments}

I would like to thank Brendan O'Connor for his comments on an early draft of this piece.

\section{Conflict of Interest and Funding}

No conflict of interest or specific source of funding.

\section{Author Contributions}

I am the sole author of the article.

\section{Open Science Practices}

This article is a theoretical article that was not preregistered nor had data or materials to share. The entire editorial process, including the open reviews, are published in the online supplement.

\section{References}

Ahuja, N. K. (2013). "It Feels Good To Be Measured" clinical role-play, Walker Percy, and the tingles. Perspectives in Biology and Medicine, 56(3), 442-451. https://doi.org/10.1353/pbm.2013. 0022

Anderson, S. F., Kelley, K., \& Maxwell, S. E. (2017). Sample-Size Planning for More Accurate Statistical Power: A Method Adjusting Sample Effect Sizes for Publication Bias and Uncertainty. Psychological Science, 28(11), 1547-1562. https:// doi.org/10.1177/0956797617723724

Bakker, M., Hartgerink, C. H. J., Wicherts, J. M., \& van der Maas, H. L. J. (2016). Researchers' Intuitions About Power in Psychological Research. Psychological Science, 27(8), 1069-1077. https: //doi.org/10.1177/0956797616647519

Barratt, E. L., \& Davis, N. J. (2015). Autonomous Sensory Meridian Response (ASMR): A flow-like mental state. PeerJ, 3, e851. https://doi.org/ $10.7717 /$ peerj.851

Barratt, E. L., Spence, C., \& Davis, N. J. (2017). Sensory determinants of the autonomous sensory meridian response (ASMR): Understanding the triggers. PeerJ, 5. https:// doi.org/10.7717/ peerj.3846

Bedwell, S., \& Butcher, I. (2020). The Co-occurrence of Alice in Wonderland Syndrome and Autonomous Sensory Meridian Response. PsyPag Quarterly, 114(1), 18-26. https://doi.org/http: //www.open-access.bcu.ac.uk/id/eprint/8749

Boag, S., Roberts, N., \& Beath, A. (2021). Commentary on Hostler: On collaboration and converging methods for rigorous Autonomous Sensory Meridian Response research.
Camerer, C. F., Dreber, A., Holzmeister, F., Ho, T.-H., Huber, J., Johannesson, M., Kirchler, M., Nave, G., Nosek, B. A., Pfeiffer, T., Altmejd, A., Buttrick, N., Chan, T., Chen, Y., Forsell, E., Gampa, A., Heikensten, E., Hummer, L., Imai, T., ... $\mathrm{Wu}, \mathrm{H}$. (2018). Evaluating the replicability of social science experiments in Nature and Science between 2010 and 2015. Nature Human Behaviour, 2(9), 637-644. https://doi.org/10. 1038/s41562-018-0399-z

Cash, D. K., Heisick, L. L., \& Papesh, M. H. (2018). Expectancy effects in the Autonomous Sensory Meridian Response. PeerJ, 6. https://doi.org/ 10.7717/peerj.5229

Chambers, C. (2017). The Seven Deadly Sins of Psychology: A Manifesto for Reforming the Culture of Scientific Practice. Princeton University Press. https://doi.org/10.2307/j.ctvc779w5

Chambers, C., \& Tzavella, L. (2020). Registered Reports: Past, Present and Future (Preprint) [Type: preprint]. MetaArXiv. https://doi.org/ 10.31222/osf.io/43298

[Accessed 26/03/2020]

Clyburne-Sherin, A., Fei, X., \& Green, S. A. (2018). Computational Reproducibility via Containers in Social Psychology (Preprint). PsyArXiv. https: //doi.org/10.31234/osf.io/mf82t [Accessed 26/03/2020]

Ebersole, C. R., Atherton, O. E., Belanger, A. L., Skulborstad, H. M., Allen, J. M., Banks, J. B., Baranski, E., Bernstein, M. J., Bonfiglio, D. B., Boucher, L., Brown, E. R., Budiman, N. I., Cairo, A. H., Capaldi, C. A., Chartier, C. R., Chung, J. M., Cicero, D. C., Coleman, J. A., Conway, J. G., .. Nosek, B. A. (2016). Many Labs 3: Evaluating participant pool quality across the academic semester via replication. Journal of Experimental Social Psychology, 67, 68-82. https://doi.org/10.1016/j.jesp.2015.10.012

Ferguson, C. J., \& Heene, M. (2012). A Vast Graveyard of Undead Theories: Publication Bias and Psychological Science's Aversion to the Null. Perspectives on Psychological Science, 7(6), 555-561. https : / / doi . org / 10 . 1177/ 1745691612459059

Fredborg, B., Clark, J., \& Smith, S. (2017). An Examination of Personality Traits Associated with Autonomous Sensory Meridian Response (ASMR). FRONTIERS IN PSYCHOLOGY, 8. https:// doi. org/10.3389/fpsyg.2017.00247

Fredborg, B., Clark, J., \& Smith, S. (2018). Mindfulness and autonomous sensory meridian re- 
sponse (ASMR). PeerJ, 6. https://doi.org/10. $7717 /$ peerj.5414

Gelman, A., \& Loken, E. (2019). The garden of forking paths : Why multiple comparisons can be a problem, even when there is no "fishing expedition " or " p-hacking" and the research hypothesis was posited ahead of time.

Giner-Sorolla, R. (2012). Science or Art? How Aesthetic Standards Grease the Way Through the Publication Bottleneck but Undermine Science. Perspectives on Psychological Science, 7(6), 562-571. https : / / doi . org / 10 . 1177/ 1745691612457576

Hambrick, D. C., \& Chen, M.-J. (2008). New Academic Fields as Admittance-Seeking Social Movements: The Case of Strategic Management. The Academy of Management Review, 33(1), 32-54. https://doi.org/10.2307/20159375

Hardwick, J. (2022). Top YouTube Searches (as of May 2022) [Publication Title: ahrefsblog]. Retrieved May 17, 2020, from https://ahrefs.com/blog/ top-youtube-searches/

Higginson, A. D., \& Munafò, M. R. (2016). Current Incentives for Scientists Lead to Underpowered Studies with Erroneous Conclusions. PLOS Biology, 14(11), e2000995. https://doi.org/10. 1371/journal.pbio.2000995

Holcombe, A. O. (2019). Contributorship, Not Authorship: Use CRediT to Indicate Who Did What. Publications, 7(3), 48. https:// doi .org / 10 . 3390/publications7030048

Hostler, T. J., Poerio, G. L., \& Blakey, E. (2019). Still More Than a Feeling: Commentary on Cash et al., "Expectancy Effects in the Autonomous Sensory Meridian Response" and Recommendations for Measurement in Future ASMR Research. Multisensory Research, 32(6), 521-531. https://doi.org/10.1163/22134808-20191366

Houtkoop, B. L., Chambers, C., Macleod, M., Bishop, D. V. M., Nichols, T. E., \& Wagenmakers, E.-J. (2018). Data Sharing in Psychology: A Survey on Barriers and Preconditions. Advances in Methods and Practices in Psychological Science, 1(1), 70-85. https : / / doi . org / 10 . 1177/ 2515245917751886

Huba, G. J., Singer, J. L., Aneshensel, C. S., \& Antrobus, J. S. (1982). Manual for the Short Imaginal Processes Inventory. Research Psychologist Press.

Ioannidis, J. P. A. (2005). Why Most Published Research Findings Are False. PLoS Medicine, 2(8), e124. https : / / doi . org / 10 . 1371 / journal . pmed . 0020124
Keizer, A., Chang, T. H. (, O’Mahony, C. J., Schaap, N. S., \& Stone, K. D. (2020). Individuals Who Experience Autonomous Sensory Meridian Response Have Higher Levels of Sensory Suggestibility. Perception, 49(1), 113-116. https:// doi.org/ $10.1177 / 0301006619891913$

Klein, O., Hardwicke, T. E., Aust, F., Breuer, J., Danielsson, H., Hofelich Mohr, A., Ijzerman, H., Nilsonne, G., Vanpaemel, W., \& Frank, M. C. (2018). A Practical Guide for Transparency in Psychological Science. Collabra: Psychology, 4(1), 20. https://doi.org/10.1525/collabra. 158

Klein, R. A., Ratliff, K. A., Vianello, M., Adams, R. B., Bahník, Š., Bernstein, M. J., Bocian, K., Brandt, M. J., Brooks, B., Brumbaugh, C. C., Cemalcilar, Z., Chandler, J., Cheong, W., Davis, W. E., Devos, T., Eisner, M., Frankowska, N., Furrow, D., Galliani, E. M., ... Nosek, B. A. (2014). Investigating Variation in Replicability: A "Many Labs" Replication Project. Social Psychology, 45(3), 142-152. https : / / doi . org / 10 . 1027 / 1864 9335/a000178

Klein, R. A., Vianello, M., Hasselman, F., Adams, B. G., Adams, R. B., Alper, S., Aveyard, M., Axt, J. R., Babalola, M. T., Bahník, Š., Batra, R., Berkics, M., Bernstein, M. J., Berry, D. R., Bialobrzeska, O., Binan, E. D., Bocian, K., Brandt, M. J., Busching, R., ... Nosek, B. A. (2018). Many Labs 2: Investigating Variation in Replicability Across Samples and Settings. Advances in Methods and Practices in Psychological Science, 1(4), 443-490. https : / / doi .org / 10 .1177/ 2515245918810225

Kvarven, A., Strømland, E., \& Johannesson, M. (2019). Comparing meta-analyses and preregistered multiple-laboratory replication projects. Nature Human Behaviour. https://doi.org/10.1038/ s41562-019-0787-z

[Accessed 26/03/2020]

Lakatos, I. (1978). The Methodology of Scientific Research Programmes: Philosophical Papers [Publication Title: Cambridge Core]. https :/ / doi . org/10.1017/CBO9780511621123

Lakens, D. (2013). Calculating and reporting effect sizes to facilitate cumulative science: A practical primer for t-tests and ANOVAs. Frontiers in Psychology, 4. https://doi.org/10.3389/fpsyg. 2013.00863

Lee, M., Song, C.-B., Shin, G.-H., \& Lee, S.-W. (2019). Possible Effect of Binaural Beat Combined With Autonomous Sensory Meridian Response for Inducing Sleep. Frontiers in Human Neuroscience, 
13. https:// doi.org/10.3389/fnhum. 2019. 00425

Liu, M., \& Zhou, Q. (2019). A Preliminary Compilation of a Digital Video Library on Triggering Autonomous Sensory Meridian Response (ASMR): A Trial Among 807 Chinese College Students. FRONTIERS IN PSYCHOLOGY, 10. https://doi. org/10.3389/fpsyg.2019.02274

Lochte, B. C., Guillory, S. A., Richard, C. A. H., \& Kelley, W. M. (2018). An fMRI investigation of the neural correlates underlying the autonomous sensory meridian response (ASMR). Bioimpacts, 8(4), 295-304. https:// doi .org/10.15171/ bi. 2018.32

Maxwell, S. E. (2004). The Persistence of Underpowered Studies in Psychological Research: Causes, Consequences, and Remedies. Psychological Methods, 9(2), 147-163. https:// doi . org/10.1037/1082-989X.9.2.147

McErlean, A. B. J., \& Banissy, M. J. (2017). Assessing Individual Variation in Personality and Empathy Traits in Self-Reported Autonomous Sensory Meridian Response. Multisensory Research, 30(6, SI), 601-613. https://doi.org/10.1163/ 22134808-00002571

McErlean, A. B. J., \& Banissy, M. J. (2018). Increased misophonia in self-reported Autonomous Sensory Meridian Response. PeerJ, 6. https://doi. org/10.7717/peerj.5351

McErlean, A. B. J., \& Osborne-Ford, E. J. (2020). Increased absorption in autonomous sensory meridian response. PeerJ, 8, e8588. https://doi. org/10.7717/peerj. 8588

Merton, R. K. (1974). The Sociology of Science: Theoretical and Empirical Investigations (1st ed.). University of Chicago Press.

Meyer, M. N. (2018). Practical Tips for Ethical Data Sharing. Advances in Methods and Practices in Psychological Science, 1(1), 131-144. https:// doi.org/10.1177/2515245917747656

Moshontz, H., Campbell, L., Ebersole, C. R., IJzerman, H., Urry, H. L., Forscher, P. S., Grahe, J. E., McCarthy, R. J., Musser, E. D., Antfolk, J., Castille, C. M., Evans, T. R., Fiedler, S., Flake, J. K., Forero, D. A., Janssen, S. M. J., Keene, J. R., Protzko, J., Aczel, B., ... Chartier, C. R. (2018). The Psychological Science Accelerator: Advancing Psychology Through a Distributed Collaborative Network. Advances in Methods and Practices in Psychological Science, 1(4), 501-515. https://doi.org/10.1177/2515245918797607

Munafò, M. R., Nosek, B. A., Bishop, D. V. M., Button, K. S., Chambers, C., Percie du Sert, N., Simon- sohn, U., Wagenmakers, E.-J., Ware, J. J., \& Ioannidis, J. P. A. (2017). A manifesto for reproducible science. Nature Human Behaviour, 1(1). https://doi.org/10.1038/s41562-016-0021

Nosek, B. A., Ebersole, C. R., DeHaven, A. C., \& Mellor, D. T. (2018). The preregistration revolution. Proceedings of the National Academy of Sciences, 115(11), 2600-2606. https://doi.org/ $10.1073 /$ pnas. 1708274114

Open Science Collaboration. (2015). Estimating the reproducibility of psychological science. Science, 349(6251), aac4716-aac4716. https : / / doi . org/10.1126/science.aac4716

Poerio, G. L., Blakey, E., Hostler, T. J., \& Veltri, T. (2018). More than a feeling: Autonomous sensory meridian response (ASMR) is characterized by reliable changes in affect and physiology (J. E. Aspell, Ed.). PLOS One, 13(6), e0196645. https://doi.org/10.1371/journal. pone.0196645

Poerio, G. L., Mank, S., \& Hostler, T. J. (2022). The Awesome as Well as the Awful: Heightened Sensory Sensitivity Predicts the Presence and Intensity of Autonomous Sensory Meridian Response (ASMR). Journal of Research in Personality, 97. https://doi.org/10.1016/j.jrp.2021.104183

Roberts, N., Beath, A., \& Boag, S. (2019). Autonomous sensory meridian response: Scale development and personality correlates. Psychology of Consciousness: Theory, Research, and Practice, 6(1), 22-39. https://doi.org/10.1037/cns0000168

Sarabipour, S., Debat, H. J., Emmott, E., Burgess, S. J., Schwessinger, B., \& Hensel, Z. (2019). On the value of preprints: An early career researcher perspective. PLOS Biology, 17(2), e3000151. https : / / doi . org / 10 . 1371 / journal . pbio . 3000151

Savage, C. J., \& Vickers, A. J. (2009). Empirical Study of Data Sharing by Authors Publishing in PLoS Journals (C. Mavergames, Ed.). PLOS One, 4(9), e7078. https://doi.org/10.1371/journal. pone. 0007078

Smaldino, P. E., \& McElreath, R. (2016). The natural selection of bad science. Royal Society Open Science, 3(9), 160384. https://doi.org/10.1098/ rsos. 160384

Smith, S. D., Fredborg, B. K., \& Kornelsen, J. (2019a). A functional magnetic resonance imaging investigation of the autonomous sensory meridian response. PeerJ, 7. https:// doi .org/10.7717/ peerj. 7122

Smith, S. D., Fredborg, B. K., \& Kornelsen, J. (2019b). Atypical Functional Connectivity As- 
sociated with Autonomous Sensory Meridian Response: An Examination of Five Resting-State Networks. Brain Connectivity, 9(6), 508-518. https://doi.org/10.1089/brain.2018.0618

Smith, S. D., Katherine Fredborg, B., \& Kornelsen, J. (2017). An examination of the default mode network in individuals with autonomous sensory meridian response (ASMR). Social Neuroscience, 12(4), 361-365. https://doi.org/10. 1080/17470919.2016.1188851

Speidel, R., \& Spitzer, M. (2018). Preprints: The What, The Why, The How. Retrieved March 25, 2020, from https://cos.io/blog/preprints-what-whyhow/

[Accessed 26/03/2020]

Vines, T. H., Albert, A. Y., Andrew, R. L., Débarre, F., Bock, D. G., Franklin, M. T., Gilbert, K. J., Moore, J.-S., Renaut, S., \& Rennison, D. J. (2014). The Availability of Research Data Declines Rapidly with Article Age. Current Biology, 24(1), 94-97. https://doi.org/10.1016/j.cub. 2013.11.014

Wicherts, J. M., Bakker, M., \& Molenaar, D. (2011). Willingness to Share Research Data Is Related to the
Strength of the Evidence and the Quality of Reporting of Statistical Results (R. E. Tractenberg, Ed.). PLOS One, 6(11), e26828. https://doi. org/10.1371/journal.pone.0026828

Wicherts, J. M., Borsboom, D., Kats, J., \& Molenaar, D. (2006). The poor availability of psychological research data for reanalysis. American Psychologist, 61(7), 726-728. https://doi.org/10.1037/ 0003-066X.61.7.726

Witte, E. H., \& Zenker, F. (2017). From Discovery to Justification: Outline of an Ideal Research Program in Empirical Psychology. Frontiers in Psychology, 8, 1847. https://doi.org/10.3389/fpsyg.2017. 01847

Zickfeld, J. H., Schubert, T. W., Seibt, B., Blomster, J. K., Arriaga, P., Basabe, N., Blaut, A., Caballero, A., Carrera, P., Dalgar, I., Ding, Y., Dumont, K., Gaulhofer, V., Gračanin, A., Gyenis, R., Hu, C.-P., Kardum, I., Lazarević, L. B., Mathew, L., ... Fiske, A. P. (2019). Kama muta: Conceptualizing and measuring the experience often labelled being moved across 19 nations and 15 languages. Emotion, 19(3), 402-424. https:// doi.org/10.1037/emo0000450 